\title{
Traumatismo dento-alveolar: uma visão geral sobre aspectos epidemiológicos, etiológicos, abordagem clínico- terapêutica e classificação
}

Dental-alveolar traumatism: an overview on epidemiological, ethological, clinical-therapeutic approach and classification

Trauma dento-alveolar: una visión general de los aspectos epidemiológicos, etiológicos, abordaje clínico-terapéutico y clasificación

\section{Resumo}

Introdução: Os traumatismos dento-alveolares (TDA) podem ser definidos como lesões que são causadas por impactos nos dentes e/ou tecidos duros e moles no interior e/ou exterior da cavidade bucal. Objetivo: Realizar uma revisão de literatura revisão de literatura acerca dos traumatismos dento-alveolares. Serão abordados, de forma geral, os principais índices epidemiológicos, etiologia, formas de diagnóstico e classificação dos traumatismos dentoalveolares. Métodos: Realizou-se uma busca bibliográfica na base de dados PubMed/ Medline, Scielo e Google acadêmico, tendo como filtro publicações dos últimos 10 anos. A busca foi realizada com descritores previamente selecionados. A pesquisa ocorreu em 3 fases e os artigos foram incluídos obedecendo aos critérios de inclusão e exclusão. Resultados: As lesões por TDA podem acometer crianças, adolescentes e adultos, porém, são bastante comuns durante a infância. Os dados sobre a prevalência variam e as causas mais frequentes são quedas, acidentes esportivos (ciclismo), acidentes de trânsito e violência física. A conduta realizada nesses casos interfere diretamente nos resultados do tratamento, por isso, é importante que o cirurgião-dentista possua conhecimentos adequados sobre como agir frente a essas lesões. Conclusão: Os traumatismos dento-alveolares são lesões vistas rotineiramente na prática clínica odontológica. A realização de um adequado diagnóstico é fundamental para que o tratamento seja efetivo e o paciente apresente o melhor prognóstico possível.

Palavras-chave: Traumatismo dentário; Epidemiologia; Diagnóstico; Tratamento.

\begin{abstract}
Introduction: Dentoalveolar injuries (ADD) can be defined as injuries that are caused by impacts on teeth and / or hard and soft tissues inside and / or outside the oral cavity. Objective: To perform a literature review literature review about dentoalveolar injuries. In general, the main epidemiological indices, etiology, forms of diagnosis and classification of dentoalveolar injuries will be addressed. Methods: A bibliographic search was performed in the PubMed database, filtering publications from the last 10 years. The search was performed with previously selected descriptors. The research took place in 3 phases and the articles were included according to the inclusion and exclusion criteria. Results: ADD injuries can affect children, adolescents and adults, but are quite common during childhood. Prevalence data vary and the most frequent causes are falls, sports accidents (cycling), traffic accidents and physical violence. The conduct performed in these cases directly interferes with the treatment results, so it is important that the dentist has adequate knowledge on how to act in front of these injuries. Conclusion: Dentoalveolar injuries are injuries routinely seen in clinical dental practice. Making a proper diagnosis is essential for effective treatment and for the patient to have the best possible prognosis.
\end{abstract}

Keywords: Dental trauma; Epidemiology; Diagnosis; Treatment. 


\begin{abstract}
Resumen
Introducción: Los traumatismos dento-alveolares (TDA) se pueden definir como lesiones que son causadas por impactos en dientes y / o tejidos duros y blandos dentro y / o fuera de la cavidad bucal. Objetivo: Realizar una revisión de la literatura sobre el trauma dentoalveolar. En general, se abordarán los principales índices epidemiológicos, etiología, formas de diagnóstico y clasificación de los traumatismos dento-alveolares. Métodos: Se realizó una búsqueda bibliográfica en las bases de datos académicas PubMed / Medline, Scielo y Google, utilizando como filtro publicaciones de los últimos 10 años. La búsqueda se realizó con descriptores previamente seleccionados. La investigación se desarrolló en 3 fases y los artículos se incluyeron según los criterios de inclusión y exclusión. Resultados: Las lesiones por TDA pueden afectar a niños, adolescentes y adultos, sin embargo, son bastante comunes durante la niñez. Los datos de prevalencia varían y las causas más frecuentes son las caídas, los accidentes deportivos (ciclismo), los accidentes de tráfico y la violencia física. La conducta realizada en estos casos interfiere directamente con los resultados del tratamiento, por lo que es importante que el odontólogo tenga los conocimientos adecuados sobre cómo actuar ante estas lesiones. Conclusión: Los traumatismos dentoalveolares son lesiones habituales en la práctica clínica odontológica. Hacer un diagnóstico adecuado es fundamental para que el tratamiento sea eficaz y para que el paciente presente el mejor pronóstico posible.
\end{abstract}

Palabras clave: Trauma dental; Epidemiología; Diagnóstico; Tratamiento.

\title{
1. Introdução
}

Os traumatismos dento-alveolares (TDA) podem ser definidos como lesões que são causadas por impactos nos dentes e/ou tecidos duros e moles no interior e/ou exterior da cavidade bucal. Acontecem geralmente de forma súbita, inesperada e acidental, sendo necessária uma atenção emergencial aos pacientes acometidos (Lam, 2016).

Os TDA podem acometer crianças, adolescentes e adultos, porém são bastante comuns durante a infância, ocorrendo com bastante frequência em crianças de até 12 anos de idade (Lima et al., 2017). Representam um problema estético-funcional e psicológico, envolvendo o emocional tanto do paciente acometido como de seus familiares (Lars, 2013).

Os dados sobre a prevalência variam entres os diferentes países, grupos etários, sexos, condições socioeconômicas e ambientais. As diferentes prevalências observadas podem estar relacionadas às discrepâncias dos procedimentos de amostragem e condução metodológica das pesquisas realizadas e a falta de sistemas padronizados de registro e classificação de TDA observados na literatura (Petti et al., 2018).

As causas mais frequentes são quedas, acidentes esportivos (ciclismo), acidentes de trânsito e violência física (Cervantes \& Torres, 2019; Zaleckiene et al., 2014). Além disso, outros fatores também podem estar relacionados e atuarem como fator predisponente, são eles: cobertura labial inadequada da parte superior dos dentes anteriores e ausência de protetores bucais durante a prática de esportes (Lam, 2016).

De maneira geral, apenas um dente é afetado, porém, atividades esportivas, violência física e acidentes de trânsito podem resultar em múltiplas lesões (Guedes Pinto et al., 2016; Zaleckiene et al., 2014).

A conduta realizada nesses casos interfere diretamente nos resultados do tratamento. Por esse motivo, é importante que não só o cirurgião-dentista (CD) possua conhecimentos e habilidades para a condução das lesões, mas também, os pais, professores e treinadores esportivos devem possuir conhecimentos básicos sobre como agir frente à esses tipos de emergências (Zaleckiene et al., 2014).

$\mathrm{Na}$ abordagem inicial é importante observar os seguintes aspectos: condições dos tecidos de suporte dentários e elementos adjacentes, uso de medicação sistêmica, grau de desenvolvimento radicular do elemento traumatizado, manobras de redução a serem utilizadas, tempo decorrido do trauma até o atendimento e aplicação de fixação quando necessário. Ainda há bastante discordância entre pesquisadores e autores sobre qual o melhor caminho a ser seguido na conduta frente a esses traumatismos (Prado \& Salim, 2018).

Dessa forma, o presente estudo tem como objetivo realizar uma revisão de literatura acerca dos traumatismos dentoalveolares. Serão abordados, de forma geral, os principais índices epidemiológicos, etiologia, formas de diagnóstico e classificação. 


\section{Metodologia}

Este estudo caracterizou-se por uma busca bibliográfica na base de dados PubMed / Medline, Scielo e Google acadêmico. A pesquisa foi realizada e teve como filtro a publicação dos últimos 10 anos. Também foram adicionados alguns artigos anteriores aos últimos 10 anos considerados relevantes para este estudo. Os descritores utilizados para busca foram: Traumatic dental injuries, Luxations teeth e Injuries alveolar process.

A pesquisa ocorreu em três fases:

1 Busca nas bases de dados com os descritores selecionados;

2 Leitura dos resumos e definição dos artigos a serem incluídos;

3 Leitura dos artigos na íntegra e construção dos resultados.

Como critérios de inclusão, foram adotados os artigos escritos em inglês e português, aqueles que se enquadravam no enfoque do trabalho e os mais relevantes em termos de delineamento das informações desejadas. Dentre os critérios observados para a escolha dos artigos foram considerados os seguintes aspectos: disponibilidade do texto integral do estudo e clareza no detalhamento metodológico utilizado. Alguns livros considerados relevantes para este estudo foram adicionados.

Foram excluídos da amostra os artigos que não apresentavam relevância clínica sobre o tema abordado, não condizentes com o assunto, artigos não disponíveis e artigos duplicados.

\section{Revisão de Literatura}

\subsection{Epidemiologia}

Os traumatismos dento-alveolares (TDA) podem ser considerados um problema de saúde pública. Dentre todas os traumas orais, são os que ocorrem com mais frequência na população (92\%), seguidos por lesões dos tecidos moles bucofaciais (28\%), já as fraturas dos ossos maxilofaciais representam apenas 6\% (Lars, 2013; Pogrel et al., 2016).

As lesões aos tecidos de suporte (luxação extrusiva, luxação intrusiva, luxação lateral, luxação e avulsão dental) compreendem 15 a $61 \%$ dos TDA e são considerados mais severas pois podem predispor a complicações em até anos após o acidente traumático (Lima et al., 2015).

Crianças na faixa etária entre 8 e 12 anos são mais acometidas sendo os dentes permanentes os mais atingidos (Cervantes e Torres, 2019; Prado e Salim, 2018). Nos estudos realizados no Brasil, a prevalência dos TDA em crianças em idade pré-escolar varia de 9,4 a 36\%. Na literatura mundial, esse percentual apresenta variação de 16,6\% a 35\% (Pogrel et al., 2016).

O traumatismo em dentes decíduos pode ser o primeiro contato da criança com o dentista. De forma que, a procura por atendimento pode ser em hospitais, postos de saúde (emergências) e consultórios particulares (Guedes Pinto et al., 2016).

De acordo com Brullmann et al. (2011) os dados sobre a frequência de TDA em dentes anteriores entre as idades de 6 e 17 anos tendem a variar muito na literatura. As prevalências nessa faixa etária nos seus estudos transversais foram entre $6,4 \%$ e $37,9 \%$.

Petti et al. (2018) realizou um estudo com o objetivo fornecer estimativas confiáveis da prevalência de TDA e taxas de incidência em dentes decíduos e permanentes. Nesse estudo, os autores concluíram que mais de um bilhão de pessoas tiveram TDA. Após a cárie dentária é a segunda doença bucal mais frequente. Entretanto, de acordo com Andreasen et al. (2012) é provável que o mundo hoje tenha mais de 3 bilhões de vítimas de TDA e a este número sejam adicionados 60 milhões de novos pacientes a cada ano. 
A maioria dos TDA envolve os dentes anteriores tanto na dentição decídua quanto na dentição permanente. Os dentes acometidos com maior frequência são os incisivos centrais superiores, seguido pelos incisivos laterais superiores, incisivos centrais inferiores, incisivos laterais inferiores e caninos superiores e inferiores. $\mathrm{O}$ acometimento aos dentes posteriores (prémolares e molares) são mais raros (Guedes Pinto et al., 2016; Lam, 2016; Zaleckiene et al., 2014).

Estudos demonstram que homens são duas vezes mais acometidos do que as mulheres. Isto pode ser atribuído à uma maior participação do gênero masculino em esportes de contato, lutas e acidentes (Guedes pinto et al., 2016; Lam, 2016; Zaleckiene et al., 2014).

Na dentição decídua é mais comum a ocorrência de lesões por luxação e na dentição permanente fraturas da coroa são mais comumente relatadas. Dessas, a fratura de esmalte é a mais frequente, seguida pela fratura de esmalte e dentina (Diangelis et al., 2012).

\subsection{Fatores etiológicos e predisponentes}

Os TDA apresentam diversos fatores etiológicos, que podem envolver questões ambientais (parques inseguros) e individuais (aumento da protusão e overjet, déficit de atenção, hiperatividade, limitações físicas). Foi observado que os fatores etiológicos relacionados aos TDA em crianças permanecem os mesmos ao longo dos anos (Kirzioglu e Oz, 2019). Esses fatores etiológicos podem, também, estar muito relacionados à idade do paciente. Por exemplo, em crianças de idade préescolar as causas mais comuns são quedas, já em crianças de idade escolar os traumatismos apresentam como principal fator etiológico os esportes de contato. Ao contrário, no grupo de adultos jovens, as lesões são frequentemente associadas aos acidentes de trânsito (Lars, 2013).

É relatado também na literatura as lesões dentárias sofridas por pacientes epilépticos que ocorrem durante as convulsões, também em pacientes com deficiência mental e usuários de drogas ilícitas (Guedes Pinto et al., 2016). Outras causas podem incluir o trauma direto, onde os dentes são atingidos diretamente por um objeto (Moule \& Cohenca, 2016).

Fatores anatômicos também podem facilitar a ocorrência de lesões traumáticas como os pacientes com prognatismo mandibular ou classe II com divisão 1 de Angle, agenesia dos incisivos laterais, inserção labial baixa e apinhamento dentário (Prado \& Salim, 2018; Baratieri et al., 2015).

Além disso, cobertura inadequada dos lábios e overjet acentuados também são fatores predisponentes (Freire et al., 2014; Lima et al., 2017). Em um estudo realizado por Côrrea-Faria et al. (2015) com 301 crianças com idade entre um e cinco anos foi possível observar que a maioria das crianças com cobertura labial inadequada tinha pelo menos um dente fraturado e aquelas que apresentavam um overjet acentuado também possuíam maior frequência de TDA.

De acordo com Sigurdsson (2013) a principal forma de prevenção para essas lesões é educando os pais, responsáveis, professores, funcionários e até mesmo as crianças e adolescentes sobre como evitá-las e o que fazer caso ocorra uma lesão. Além de que, é importante que todo dentista discuta com os pacientes durante as consultas odontológicas de rotina, os fatores de risco que podem levar às lesões por traumatismo dentário. Sendo assim, os profissionais devem identificar e orientar os pacientes que possuem elevado risco de sofrer traumatismos dentários especialmente os que praticam esportes, para que atentem à importância do uso de protetores bucais.

\subsection{Diagnóstico}

Em situações de TDA principalmente em crianças, é importante que o CD seja capaz de transmitir tranquilidade e confiança ao paciente e seus acompanhantes. Muitas vezes, esta situação traumática pode representar o primeiro contato da criança com o dentista (Guedes Pinto et al., 2016). 


\subsubsection{Anamnese}

Inicialmente, deve-se realizar uma anamnese detalhada obtendo-se do paciente uma história completa do acidente para um adequado diagnóstico. É importante que sejam feitas diversas perguntas sobre como ocorreu o traumatismo, a Tabela 1 descrita abaixo exemplifica algumas dessas perguntas (Hupp et al., 2015).

Tabela 1 - Resumo das perguntas a serem realizadas durante a anamnese e sua importância.

\begin{tabular}{|c|c|}
\hline Quem é o paciente? & $\begin{array}{l}\text { Nome, endereço, número de telefone e outros } \\
\text { dados demográficos. }\end{array}$ \\
\hline Quando ocorreu a lesão? & $\begin{array}{l}\text { O prognóstico dos tratamentos de dentes com } \\
\text { mobilidade, fraturas coronárias e fraturas } \\
\text { alveolares podem ser influenciadas pela demora } \\
\text { no tratamento. }\end{array}$ \\
\hline Onde ocorreu a lesão? & $\begin{array}{l}\text { Avaliar o grau de contaminação bacteriana } \\
\text { dependendo do local onde aconteceu o } \\
\text { traumatismo. }\end{array}$ \\
\hline Como aconteceu a lesão? & $\begin{array}{l}\text { Investigar a possibilidade de outras lesões, } \\
\text { informações que podem levar à causa real do } \\
\text { traumatismo, condições clínicas preexistentes } \\
\text { (convulsão). }\end{array}$ \\
\hline $\begin{array}{l}\text { Qual o tratamento realizado desde que a } \\
\text { lesão ocorreu? (se houve algum) }\end{array}$ & $\begin{array}{l}\text { Como o dente foi armazenado em casos de } \\
\text { avulsão, se houve reimplante de um dente } \\
\text { parcialmente avulsionado. }\end{array}$ \\
\hline $\begin{array}{l}\text { Alguém observou dentes ou fragmentos de } \\
\text { dentes no local do acidente? }\end{array}$ & $\begin{array}{l}\text { Podem ser necessários exames radiográficos dos } \\
\text { tecidos moles periorais, do tórax e da região } \\
\text { abdominal para descartar a presença de } \\
\text { fragmentos dentários no interior dos tecidos } \\
\text { moles ou outras cavidades do corpo. }\end{array}$ \\
\hline Qual o estado de saúde geral do paciente? & $\begin{array}{l}\text { Obter uma sucinta história clínica do paciente } \\
\text { (alergias medicamentosas, sopro cardíaco, } \\
\text { discrasias sanguíneas ou outras doenças } \\
\text { sistêmicas). }\end{array}$ \\
\hline $\begin{array}{l}\text { O paciente teve náusea, vômito, } \\
\text { inconsciência, amnésia, cefaléia, distúrbios } \\
\text { visuais ou confusão após acidente? }\end{array}$ & Podem indicar traumatismo intracraniano. \\
\hline Existe alguma alteração na oclusão? & $\begin{array}{l}\text { Reposta afirmativa pode indicar luxação } \\
\text { dentária, fratura dentoalveolar ou dos maxilares. }\end{array}$ \\
\hline
\end{tabular}


Deve-se obter a história médica pregressa pessoal e familiar do paciente investigando o uso de medicamentos, histórico de alergias, hemorragias, cardiopatias, hipertensão arterial sistêmica e transtornos neurológicos, tendo em vista que todos esses fatores podem influenciar no planejamento bem como na prescrição medicamentosa do paciente acometido (Prado \& Salim, 2018).

Se a lesão aconteceu em um local ou por um objeto com alto potencial de risco de contaminação é importante realizar a administração de antibióticos e a profilaxia antitetânica, independente do seu estado de imunização (Audi e Wright, 2010; Lim \& Sirichai, 2016; Moule \& Cohenca, 2016).

O CD tem a obrigação, por lei, de relatar evidência de suspeita de abuso. Por esse motivo, é importante buscar indícios se as lesões possuem associação com algum tipo de abuso físico ou violência doméstica, principalmente em crianças e idosos. Portanto, deve-se ficar atento se o paciente possuir lesões múltiplas, em que sua distribuição não seja consistente com um único acidente ou com a descrição do incidente relatado (Moule \& Cohenca, 2016).

\subsubsection{Exame físico (intra e extraoral)}

O exame físico também é bastante importante para um correto diagnóstico. Devem ser avaliados sinais vitais como pulso, pressão arterial e frequência respiratória. É imprescindível não focar somente em uma estrutura, tendo em vista que lesões concomitantes podem estar presentes (Hupp et al., 2015; Prado \& Salim, 2018).

Inicialmente, deve-se limpar com solução salina estéril os detritos ou sangue em toda região da face e pescoço para permitir uma melhor avaliação de todas as lesões teciduais (Auld \& Wright, 2010).

A face e todas as margens ósseas, incluindo a órbita, devem ser palpadas em busca de sinais de fraturas ósseas. Devem ser examinadas visualmente e palpadas as regiões do pescoço, articulações temporomandibulares (ATMs), tecidos moles intra e extraorais, maxila, mandíbula e as regiões adjacentes que estão próximas à lesão. Alterações na oclusão podem indicar fratura dos maxilares. O registro fotográfico também é essencial para documentação do caso (Moule \& Cohenca, 2016).

Os tecidos intraorais devem ser examinados cuidadosamente. Se houverem feridas nos lábios, língua, palato e vestíbulo bucal deve-se explorar afim de investigar a presença de corpos estranho no interior desses tecidos. Além disso, devem ser observados os danos às estruturas vitais, tais como ducto parotídeo, ducto submandibular, nervos e vasos sanguíneos (Guedes Pinto et al., 2010).

As lesões traumáticas dentárias são frequentemente associadas à lesões dos tecidos moles. Suhadi (2012) relatou em seu estudo que $62,8 \%$ de todos os pacientes atendidos em um pronto-socorro hospitalar por lesões orais apresentavam laceração do lábio. É preciso ficar atento, pois, muitas vezes essas lesões podem ser negligenciadas nas situações de emergência, de forma que fragmentos de tecidos dentários podem ser deixados dentro dos tecidos moles se esses não forem corretamente inspecionados durante o exame físico.

Nesta etapa, devem ser observadas não apenas alterações que ocorreram em função do trauma atual, mas também, é importante buscar e registrar alterações prévias afim de evitar dúvidas sobre possíveis sequelas; se estas são realmente em decorrência do trauma ou já existiam previamente e não foram diagnosticadas na consulta inicial (Prado \& Salim, 2018).

O exame das coroas dentárias deve ser feito cuidadosamente observando os seguintes aspectos: presença de fraturas e a extensão da fratura, exposição pulpar, mobilidade tanto em sentido horizontal quanto na direção axial, deslocamentos, anormalidades na oclusão. Além disso, verificar se há dor durante a percussão dental, isto pode indicar dano ao ligamento periodontal (LPD) (Pogrel et al., 2016).

Alterações na cor dentária pode acontecer em dentes traumatizados. A presença de pigmentos na cor rosa ou roxa ocorrem como resultado de um sangramento na dentina, contudo, isso pode ser resolvido ao longo do tempo. Já os dentes que 
apresentam descoloração cinza ou marrom, representam um forte indício de necrose pulpar, devendo ser confirmado pelos testes de vitalidade pulpar. A longo prazo, pode ocorrer também uma calcificação na câmara pulpar e o sinal principal é a descoloração dentária na cor amarela (Moule \& Cohenca, 2016).

Outro aspecto importante a ser analisado, principalmente se o paciente estiver inconsciente, é quando houver falta de dentes, parte de dentes ou próteses. Nesses casos, a suspeita de aspiração ou ingestão deve ser considerada e devem ser solicitadas radiografias de tórax e abdome. Normalmente, quando há inalação de corpos estranhos o paciente poderá sentir sintomas como tosse (Pogrel et al., 2016).

Os testes de sensibilidade e vitalidade pulpar após os TDA são bastantes difíceis e devem ser realizados com cuidado pois resultados falsos podem aparecer. Isso acontece porque a sensibilidade das estruturas dentais imediatamente após lesões traumáticas encontra-se temporariamente ou permanentemente reduzida, especialmente após ferimentos com luxação (Pogrel et al., 2016).

A grande maioria dos autores citados defende que o teste de sensibilidade pulpar não deve ser realizado imediatamente após ao traumatismo, pois resultados falso-negativos podem aparecer. No entanto, Moule \& Cohenca (2016) argumenta que pode ser realizado o teste de sensibilidade pulpar na visita de emergência para estabelecer uma linha de base para futuros testes e acompanhamento do caso.

Ainda, segundo Moule \& Cohenca (2016) com relação aos testes de sensibilidade pulpar após o trauma, três situações são passíveis de acontecer. A primeira é a polpa não responder no momento da avaliação inicial nem nas consultas de revisão. Isso não deve ser indicativo de que ocorreu necrose pulpar, e sim, que o dente seja colocado sob revisão; a segunda, é quando um dente não responde ao teste de sensibilidade pulpar precoce, mas responde mais tarde (geralmente após 6 a 8 semanas); e a terceira é quando um dente responde positivamente ao teste de polpa na avaliação inicial, mas não responde à revisão. Este é um cenário negativo e desfavorável e, na maioria das vezes, indica que ocorreu necrose pulpar, mas que deve ser confirmado por testes adicionais e outros achados do exame clínico.

A necrose pulpar pode ser confirmada se o dente também se tornar assintomático, se houver uma mudança para uma cor cinza, se houver reabsorção inflamatória da raiz ou se for observada uma radiolucência periapical (Moule \& Cohenca, 2016). 
Tabela 2 - Resumo das áreas que devem ser examinadas e o que deve ser observado durante o exame físico de traumatismos dento-alveolares.

\begin{tabular}{|c|c|}
\hline Feridas extraorais de tecido mole & $\begin{array}{l}\text { Lacerações, abrasões e contusões. Se há alguma estrutura vital } \\
\text { cruzando a linha de laceração. }\end{array}$ \\
\hline Feridas intraorais de tecido mole & $\begin{array}{l}\text { Verificar a presença de corpos estranhos como dentes ou coroas } \\
\text { dentro das lesões de lábio, assoalho e bochechas e outras áreas. }\end{array}$ \\
\hline $\begin{array}{l}\text { Fraturas dos maxilares ou do processo } \\
\text { alveolar }\end{array}$ & $\begin{array}{l}\text { Fraturas dos maxilares é verificada por meio da palpação. } \\
\text { Segmentos fraturados do processo alveolar podem ser } \\
\text { diagnosticados por palpação e exame visual. }\end{array}$ \\
\hline Coroas dentárias & $\begin{array}{l}\text { Os dentes devem ser limpos do sangue para observar a extensão e } \\
\text { profundidade das fraturas e se há exposição pulpar. }\end{array}$ \\
\hline Deslocamento dentário & Deslocamento na direção vestíbulo-lingual, intrusão e extrusão. \\
\hline Mobilidade dentária & $\begin{array}{l}\text { Conferir a mobilidade em todos os dentes nas direções vertical e } \\
\text { horizontal. }\end{array}$ \\
\hline Percussão dentária & Determina se o ligamento periodontal sofreu algum dano. \\
\hline Testes de vitalidade pulpar & $\begin{array}{l}\text { Determinar o tipo de tratamento que os dentes receberão. } \\
\text { Resultados falso-negativos podem acontecer, sendo assim, } \\
\text { deve-se se repetir os testes novamente após semanas e antes da } \\
\text { terapia endodôntica. }\end{array}$ \\
\hline
\end{tabular}

Fonte: Adaptação (Hupp et al., 2015).

\subsubsection{Exame radiográfico}

Através da análise do exame radiográfico é possível visualizar alterações imperceptíveis clinicamente. De maneira geral, é importante buscar no exame radiográfico as seguintes informações: presença de fratura radicular, grau de extensão de intrusão ou extrusão, presença de doença periapical pré-existente, extensão do desenvolvimento radicular, presença de fratura dos maxilares e fragmentos de dentes e corpos estranhos alojados nos tecidos moles (Guedes Pinto et al., 2016; Hupp et al., 2015).

A radiografia panorâmica pode ser utilizada para uma observação geral das estruturas, como em casos de fraturas mandibulares e maxilares, porém não é tão indicada para um diagnóstico preciso dos TDA. Em decorrência disso, os dentistas podem optar por realizar radiografias periapicais principalmente nos casos de suspeita de luxações laterais, extrusivas, intrusivas, fraturas radiculares e também para avaliar o estágio de desenvolvimento radicular e a integridade da parede alveolar em casos de avulsão (Prado \& Salim, 2018).

Em alguns casos a radiografia será imprescindível para detectar com precisão qual o tipo de traumatismo que o paciente foi acometido. Por exemplo, só é possível distinguir lesões por luxação de fraturas radiculares através do exame radiográfico. Além disso, ao observarmos um alargamento do espaço periodontal podemos desconfiar de luxações extrusivas ou laterais, já em casos de dentes intruídos na maioria das vezes haverá uma falta de espaço na região do ligamento periodontal (Pogrel et al., 2016). 
Corpos estranhos que por ventura estejam dentro dos tecidos moles dos lábios ou bochechas, podem ser detectados pela realização de uma radiografia com o uso de uma película comum onde o filme é posicionado no interior dos tecidos a serem examinados, vestibularmente ao alvéolo. Para esta tomada radiográfica, o tempo de exposição deve ser reduzido à aproximadamente um terço do normal utilizado habitualmente (Hupp, et al. 2015; Pogrel, et al. 2016).

As radiografias periapicais por meio do paralelismo associadas ao exame clínico ainda é o padrão de atendimento mais utilizado e que deve ser considerado na avaliação inicial de traumatismos dentários. Contudo, algumas vezes imagens adicionais são necessárias para que se obtenha um diagnóstico preciso. As tomografias computadorizadas de feixe cônico (TCFC) devem ser consideradas, uma vez que, produzem imagens tridimensionais (3D) que permitem melhor avaliação (Cohenca \& Silberman, 2017).

Em fraturas coronorradiculares determinar precisamente a extensão apical da fratura nem sempre é possível quando se utilizam radiografias periapicais bidimensionais (2D). O uso de TCFC nesses casos é bastante recomendado. Ademais, a TCFC também pode ser bastante útil na detecção de fraturas radiculares, já que essas lesões apresentam um desafio diagnóstico em virtude das limitações de radiografias periapicais convencionais, principalmente no que se refere à superposição de estruturas anatômicas (Cohenca \& Silberman, 2017).

De acordo com Kullman e Sane (2012) a escolha ideal das radiografias deve ser individualizada para as necessidades específicas de cada paciente e, portanto, deve ser baseada no resultado da anamnese detalhada e do exame clínico. Segundo Andreasen (2015) a imagem tridimensional (TCFC) deve ser vista como um complemento à radiografia intra-oral convencional e tem desvantagens como o aumento da radiação e custos elevados. Além do mais, se a tomografia computadorizada por feixe cônico estiver disponível, ela deve ser considerada para lesões mais graves, como fraturas de coroa/raiz, raiz e alveolar, bem como todas as lesões de luxação.

\subsection{Tratamento}

De acordo com Andreasen et al. (2012) a multiplicidade de possíveis cenários de trauma e a ampla variedade de opções de tratamento torna muito difícil para os profissionais fornecer tratamento baseado em evidências e recomendar a melhor escolha de tratamento possível para o paciente. Pesquisas no mundo todo mostraram que o conhecimento sobre o tratamento mais adequado para dentes traumatizados é deficiente. Por esse motivo, é de se esperar que o tratamento esteja longe do ideal.

Akhlaghi et al. (2014) observaram em seu estudo que o conhecimento dos profissionais sobre o manejo de fratura não complicada da coroa (sem exposição pulpar), extrusão, dentes decíduos avulsionados e tempo crítico para reimplante de dentes permanentes avulsionados foram aceitáveis. No entanto, em casos de fratura complicada da coroa (com exposição pulpar), fratura radicular, intrusão, avulsão dos dentes permanentes e tempo de duração da imobilização não foi satisfatório. Isso se deve principalmente a grande complexidade e à maior controvérsia dos estudos acerca do tratamento dessas lesões.

O Guia de Trauma Dental do inglês Dental Trauma Guide é uma tentativa de mudar esta situação, tornando os conhecimentos atuais em traumatologia dentária facilmente disponíveis na internet. Este guia foi criado através da coleta durante 40 anos os registros dos pacientes no Hospital Universitário de Copenhague juntamente com os resultados de 79 estudos clínicos e de 65 estudos experimentais em animais utilizando macacos e apresenta diversas recomendações tanto para profissionais quanto para pacientes sobre como reagirem frente à situações emergenciais de TDA (Andreasen et al., 2012).

Além disso, a Associação Internacional de Traumatologia Dentária (International Association of Dental Traumatology - IADT) desenvolveu um conjunto atualizado de diretrizes para o manejo de lesões traumáticas com o objetivo de ajudar os dentistas, outros profissionais de saúde e pais ou responsáveis na tomada de decisões. As primeiras diretrizes da IADT foram publicadas no ano de 2001 e foram atualizadas em 2007. No ano de 2012, estas foram novamente reformuladas e 
publicadas na revista de traumatologia dentária e aparecem em três partes, abordando as fraturas e luxações dos dentes permanentes, avulsão dos dentes permanentes e lesões na dentição decídua (Diangelis et al., 2012).

Essas diretrizes são, geralmente, criadas para orientar os profissionais sobre qual caminho a ser seguido nas decisões de tratamento, sendo fáceis de serem entendidos, práticos e com objetivo de oferecer um melhor atendimento, haja vista que direcionam as melhores formas de diagnóstico e fornecem recomendações para o tratamento de lesões dentárias traumáticas (Malmgren, et al. 2012).

No entanto, segundo Diangelis et al. (2012) a IADT não pode e não garante resultados favoráveis com a adesão dessas diretrizes, mas acredita que o seu emprego pode trazer maiores chances de um prognóstico favorável.

Para otimizar o resultado do tratamento, o diagnóstico correto da gravidade da lesão é essencial e deve ser realizado por meio de uma detalhada anamnese, avaliação clínica e radiográfica (Kullman \& Sane, 2012). Entre as técnicas clínicas utilizadas para avaliar a extensão das lesões em dentes traumatizados pode-se destacar os testes de mobilidade, som de percussão, sensibilidade à percussão e resposta ao teste de vitalidade pulpar (Andreasen, 2015).

De maneira geral, no tratamento é importante restabelecer a forma e a função normais do aparelho mastigatório. Este deve ser realizado de forma bem coordenada por meio de uma equipe multidisciplinar onde cada um se dedica a observar de forma individualizada aspectos endodônticos, estéticos, funcionais, entre outros (Hupp et al., 2015; Prado \& Salim, 2018).

Sendo assim, o tratamento ideal pode envolver vários especialistas em odontologia como cirurgiões bucomaxilofaciais, odontopediatras, endodontistas, ortodontistas e periodontistas (Andreasen et al., 2012).

As orientações básicas que devem ser dados ao paciente que sofreu qualquer tipo de TDA são: evitar uso do dente para mastigação (não morder na região e dieta pastosa), manter a área afetada limpa através da higienização com gaze e solução à base de clorexidina (CHX) e assim que possível retornar à escovação. Avaliar a necessidade de prescrição medicamentosa (analgésico, anti-inflamatório e antibiótico) e avaliação de vacinação antitetânica, caso necessite, deve-se encaminhar ao médico (Guedes Pinto et al., 2010).

A abordagem aos traumatismos em dentes decíduos é semelhante àquelas para dentes permanentes. É importante que se realize um adequado exame clínico e radiográfico para um correto diagnóstico. Além disso, os pais e responsáveis devem receber as instruções necessárias sendo orientados da importância dos cuidados de higienização bucal e também sobre as possíveis complicações, como as que podem ocorrer no desenvolvimento dos dentes permanentes (Andersson et al., 2012).

Para Fonseca (2015) dentes decíduos com qualquer tipo de fratura coronorradicular devem ser extraídos e o tratamento com próteses deve ser instituído. Já as fraturas radiculares em dentes decíduos são incomuns antes do desenvolvimento completo da raiz. No entanto, se acontecer e o dente não apresentar mobilidade elas podem ser preservadas e espera-se o dente esfoliar. Em casos que houver mobilidade e deslocamento coronário, o dente pode ser removido, deixando-se os fragmentos apicais e esperando que estes reabsorvam fisiologicamente. O dente decíduo extruído deve ser extraído para evitar danos ao dente permanente sucessor.

Existe uma relação bastante próxima entre o ápice do dente decíduo e o germe do dente permanente subjacente. Sendo assim, malformação dentária, dentes impactados e distúrbios de erupção na dentição permanente são algumas das consequências que podem ocorrer após lesões na dentição decídua e/ou osso alveolar (Malmgren et al., 2012). Além disso, estudos anteriores relataram que mais de 50\% dos dentes decíduos traumatizados apresentam modificação de cor (Hyun et al., 2016).

Qassen et al. (2014) realizou um estudo com objetivo de determinar os tipos de sequelas resultantes de luxação intrusiva e lesões subluxativas em dentes anteriores decíduos bem como o tempo de tais sequelas. 52 crianças, com setenta dentes intrusos e 76 crianças, com 99 dentes subluxados foram incluídas no estudo. Realizou-se um estudo retrospectivo longitudinal onde foram coletados dados de prontuários e radiografias de pacientes com traumatismo dentário atendidos no 
Centro de Estudos e Tratamento de Traumatologia Dentária em Odontopediatria (Pelotas, RS, Brasil). Nas lesões de subluxação, mais de 50\% dos casos de modificação de cor da coroa, cálculo pulpar, fístula e reabsorção radicular inflamatória ocorreram dentro de 180 dias após o TDA. No entanto, mesmo após o período de seguimento de 3 a 4 anos, as sequelas ainda eram diagnosticadas. Entre as sequelas de intrusão, as fístulas foram frequentemente diagnosticadas dentro de 91 a 180 dias após a lesão. A maioria dos casos de alteração de cor da coroa e calcificações pulpares foram observados nos períodos de 181 a 365 dias e 1 a 2 anos.

Não existe consenso na literatura acerca de qual o melhor tratamento para lesões traumáticas na dentição decídua. No entanto, é muito importante que o clínico tenha habilidades e experiência com pacientes pediátricos em situações de emergência (Malmgrem et al., 2012).

\subsection{Prognóstico e complicações}

De forma geral, o prognóstico é melhor em pacientes mais jovens, com tecidos moles preservados, em dentes com ausência de fraturas radiculares e com suporte ósseo íntegro (Fonseca, 2015). Além disso, depende muito da forma como é realizado o tratamento. Quanto mais cedo as lesões forem tratadas melhor será o prognóstico. Uma conduta adequada torna possível limitar os danos sofridos, bem como diminuir o posterior surgimento de sequelas (Prado \& Salim, 2018; Kirzioglu \& Oz, 2019).

As complicações causadas por TDA mais comuns incluem necrose pulpar, periodontite apical, descoloração da coroa dentária, calcificação do canal radicular, fístula, reabsorção radicular externa, recessão de tecidos moles e anquilose (Lam, 2016; Zaleckiene et al., 2014).

Segundo Lima et al. (2015) a ocorrência dessas complicações depende principalmente da severidade da lesão e também do desenvolvimento radicular. Dentes com luxação intrusiva e avulsão dental possuem mais chances de desenvolver necrose pulpar do que nos casos de luxação extrusiva e lateral.

A necrose pulpar representa a complicação mais comum em dentes em situação pós-traumática. Acontece, principalmente, nos dentes que tiveram lesões nos tecidos periodontais, sendo incomuns após fraturas de coroa, que envolvem apenas esmalte e dentina sem exposição pulpar. É preciso ficar atento ao fato de que se for diagnosticada tardiamente, a necrose pulpar pode predispor outras manifestações como a reabsorção radicular inflamatória (Elbay et al., 2014).

Segundo Hyun et al. (2016) a descoloração da coroa dentária acontece em decorrência da hemorragia intrapulpar que ocorre como resultado das lesões traumáticas. Após a hemorragia é formada hemoglobina, a partir da degradação da hemoglobina é formada hemossiderina que penetra nos túbulos dentinários alterando a cor do dente. Alguns exemplos de descoloração dos dentes podem ser vistos na figura 03 abaixo.

De acordo com Bastos \& Côrtes (2018) têm-se a hipótese de que a calcificação do canal radicular está relacionada à revascularização após lesão grave do feixe neurovascular da polpa. Ademais, não está associada apenas ao tipo de lesão, mas também à idade do paciente no momento do trauma e afeta principalmente dentes com desenvolvimento radicular incompleto no momento da lesão.

Exames periódicos são necessários para monitorar a saúde pulpar e periodontal das estruturas acometidas. Mesmo na ausência de uma lesão dentária vista clinicamente, existe um risco potencial à vitalidade pulpar em virtude do rompimento dos vasos apicais ou da hiperemia da polpa resultando em isquemia e provavelmente em necrose. Sendo assim, devem ser programadas avaliações clínicas mensais para os primeiros 6 meses que serão posteriormente sucedidas por visitas anuais ao longo de 5 anos (Fonseca, 2015). 


\subsection{Classificação dos traumatismo dento-alveolares}

Existem vários sistemas de classificação que englobam os TDA. No entanto, deve-se dar uma atenção especial ao modelo de classificação delineado pela Organização Mundial de Saúde (OMS) que surgiu em 1978 e também, ao modelo criado por Andreasen em 1982 que representa uma modificação do modelo da OMS. Este modelo delineado por Andreasen pode ser aplicado tanto para a dentição decídua quanto para a permanente (Lam, 2016; Miloro et al., 2016).

Feliciano \& Caldas (2006) realizaram uma revisão sistemática de estudos entre 1936 e 2003 e identificaram que os sistemas mais utilizados eram Andreasen (32\%), Ellis (14\%) e Garcia-Godoy (6\%). Apesar disso, a categoria 'Outros' representou 40\%, indicando uma variabilidade significativa no uso de sistemas de classificação.

O modelo delineado por Andreasen, por ser o mais utilizado na literatura, está resumido na Tabela 3.

Tabela 3 - Classificação delineada por Andreasen para as lesões traumáticas dento-alveolares.

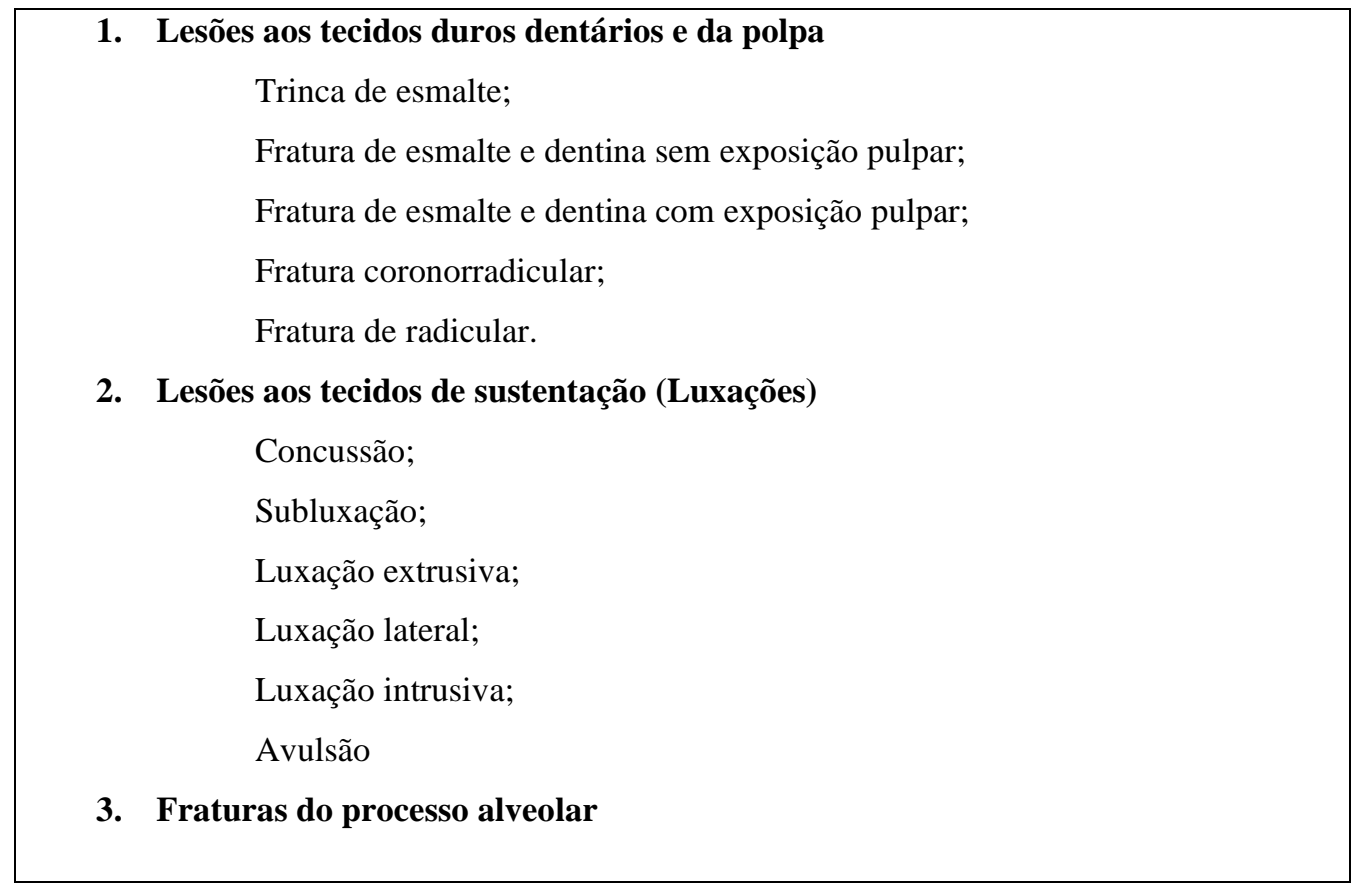

Fonte: Adaptado (Lam, 2016).

\section{Conclusão}

Os traumatismos dento-alveolares representam um grupo de lesões que ocorrem com bastante frequência nas populações, no entanto, apresentam variações de prevalência entre diferentes aspectos, como em diferentes países, grupos etários e gêneros. Acometem com maior frequência crianças de até 12 anos de idade do sexo masculino e apresentam como causas mais predominantes as quedas, acidentes esportivos, acidentes de trânsito e violência física.

Realizar um adequado diagnóstico das lesões representa uma etapa fundamental na abordagem inicial ao paciente vítima desses traumatismos. Diversos aspectos devem ser observados e registrados para que o tratamento seja efetivo e o paciente apresente um excelente prognóstico com o mínimo de complicações possíveis.

De forma geral, as lesões são classificadas em: lesões aos tecidos duros dentários e da polpa, lesões aos tecidos de suporte e lesões do processo alveolar. O paciente pode apresentar concomitantemente mais de uma lesão e a gravidade das lesões varia de acordo com as estruturas acometidas. 
Diante do exposto, é importante que o cirurgião-dentista conheça os principais fatores etiológicos, formas de diagnóstico, aspectos clínicos e de tratamento dos traumatismos dento-alveolares para que realize um manejo adequado tendo em vista que essas situações são vistas rotineiramente na prática clínica odontológica.

\section{Referências}

Akhlaghi, N. et al. (2014) General Dental Practitioners' Knowledge about the Emergency Management of Dental Trauma. Iran Endod J, 9(4), 251-256

Andersson, L. et al. (2012) International Association of Dental Traumatology guidelines for the management of traumatic dental injuries: 2. Avulsion of permanente teeth. Dent Traumatol, 28(3), 88-96.

Andreasen, J.O.; \& Lauridsen, E. (2015). Alveolar process fractures in the permanent dentition. Part 1. Etiology and clinical characteristics. A retrospective analysis of 299 cases involving 815 teeth. Dent Traumatol, 31(6), 442-447.

Auld, D. N. T.; \& Wright, G. B. (2010). The Initial Management of Dento-Alveolar Trauma in General Dental Practice. Dental Update, $37(5), 286-294$.

BaratierI, L.N. et al. (2015). Odontologia Restauradora - Fundamentos e Possibilidades. 2ed. São Paulo: Santos.

Bastos, J.V.; \& Côrtes, M.I.S. (2018). Pulp canal obliteration after traumatic injuries in permanent teeth - scientific fact or fiction? Braz. Oral. Res, 32(S75), $159-168$.

Cervantes, A.P.; \& Torres, S.C. (2019). Management of the postraumatic dental complication. A case report. Odontología Vital, $30,7-14$.

Cohenca, N.; Silberman, A. (2017). Contemporary imaging for the diagnosis and treatment of traumatic dental injuries: a review. Dent traumatol, 33(5), 321328 .

Côrrea-faria, P. et al. Influence of clinical and socioeconomic indicators on dental trauma in preschool children. Braz. Oral. Res, $29(1), 1-7$.

Dan brüllmann, D. et al. (2011). The Treatment of Anterior Dental Trauma. Dtsch Arztebl Int, 108(34-35), 565-570.

Diangelis, A. J. et al. (2012). International Association of Dental Traumatology guidelines for the management of traumatic dental injuries: 1. Fractures and luxations of permanent teeth. Dent Traumatol, 28(3), 2-12.

Elbay, U.S. et al. (2014). Multidisciplinary approach to delayed treatment of traumatic teeth injuries involving extrusive luxation, avulsion and crown fracture. Oper dent, 39(6), 566-571.

Feliciano, K. M. P. C., Caldas J. R., \& A. F. A systematic review of the diagnostic classifications of traumatic dental injuries. Dent traumatol, $22(2)$, 71-76.

Fonseca, R. J. et al. (2015). Trauma Bucomaxilofacial. 4ed. Rio de Janeiro: Elsevier.

Freire, M. C. M. et al. (2014). Association of Traumatic Dental Injuries with Individual-, Sociodemographic- and School-Related Factors among Schoolchildren in Midwest Brazil. Int J Environ Res Public Health, 11(9), 9885-9896.

Guedes pinto, A.C. (2016). Odontopediatria. 9ed. São Paulo: Santos.

Hupp, J. R.; Ellis III, E.; \&Tucker, M. R. (2015). Cirurgia oral e maxilofacial contemporânea. 6ed. Elsevier: Rio de Janeiro.

Hyun, H. K.; Shin, T.J.; Kim, Y.J. (2016). The post-traumatic colour change of primary incisors: a colourimetric and longitudinal study. Int J Paediatr Dent., 26(4), 291-300.

Kirzioglu, Z; Oz, E. (2019). Changes in the aetiological factors of dental trauma i children over time: an 18-year retrospective study. Dent traumatol, 1-9.

Kullman, L.; Sane, M.A. (2012). Guidelines for dental radiography immediately after a dento-alveolar trauma, a systematic literature review. Dent traumatol, 28(3), 193-199, 2012.

Lam, R. (2016). Epidemiology and outcomes of traumatic dental injuries: a review of the literature. Aust Dent J, 61(1), 4-20.

Lars, A. (2013). Epidemiology of traumatic dental injuries. Journal of Endodontics, 39(3), S2-S5.

Lim, L.; \& Sirichai, P. (2016). Bone fractures: assessment and management. Australian Dental Journal, 61(S.1),74-81.

Lima, T. F. R. et al. Post-traumatic Complications of Severe Luxations and replanted teeth. Dent Pratic, 16(1), 13-19.

Lima, T. F. R. et al. (2017). Relationship between Initial Attendance after Dental Trauma and Development of External Inflammatory Root Resorption. Braz. Dent. J, 28(2), 201-205.

Malmgren, B. et al. (2012). International association of dental traumatology guidelines for the management of traumatic dental injuries: 3 . injuries in the primary dentition. Dent traumatol, 28(3), 174-182.

Miloro, M. et al. (2016). Princípios de cirurgia bucomaxilofacial de Peterson. 3ed. São Paulo: Santos. 
Research, Society and Development, v. 10, n. 1, e10410111564, 2021

(CC BY 4.0) | ISSN 2525-3409 | DOI: http://dx.doi.org/10.33448/rsd-v10i1.11564

Moule, A.; \& Cohenca, N. (2016). Emergency assessment and treatment planning for traumatic dental injuries, Australian Dental Journal, 61(S1), 21-38.

Petti, S., Glendor, U.; \& Andersson, L. (2018). World traumatic dental injury prevalence and incidence, a meta-analysis—One billion living people have had traumatic dental injurie. Dental Traumatol, 34(2), 71-86.

Pogrel, M.A., Kahnberg, K. E.; \& Anderson, L. (2016). Cirurgia bucomaxilofacial. 1ed. Rio de Janeiro: Santos.

Prado, R.; \& Salim, M. (2018). Cirurgia bucomaxilofacial: diagnóstico e tratamento. 2ed. Rio de Janeiro: Guanabara Koogan.

Sigurdsson, A. (2013). Evidence-based review of prevention of dental injuries. J Endod, 39(3), S88-S93.

Zaleckiene, V., Peciuliene, V., Brukiene, V.; \& Drukteinis, S. (2014). Traumatic dental injuries: etiology, prevalence and possible outcomes. Stomatologija, Baltic Dental and Maxillofacial Journal, 16(1), 7-14. 